\title{
MODELLING OF A PERIODIC QUASI-3D ARRAY USING AN ASYMPTOTIC EXTRACTION TECHNIQUE
}

\author{
V. Volski", Y. Schols , G.A.E Vandenbosch ${ }^{*}$ \\ ${ }^{\star}$ Katholieke Universiteit Leuven, ESAT-TELEMIC, \\ Kasteelpark Arenberg 10, B-3001, Leuven, Belgium
}

Keywords: periodic array, Green's function, vertical conductors, asymptotes, acceleration of series

\begin{abstract}
An asymptotic extraction technique combined with the Method of Moments is used to model periodic quasi-3D arrays. These arrays consist of vertical and horizontal conductors. The particularities of the proposed approach are the use of special asymptotes that are used in the case of periodic arrays and single dipoles. This step facilitates at the same time the development of new features in the software code for a single dipole and a periodic array.
\end{abstract}

\section{Introduction}

In this paper we present a further development of a new asymptotic extraction technique developed to model periodic arrays. The goal of this technique is to obtain a strong resemblance between the two algorithms to calculate Green's Functions (GFs) for a single dipole and a periodic array of dipoles. The original algorithm for a single dipole is modified with a few adjustments in such a way that the periodicity of the dipoles can be implemented easily. In practice this means that almost all modelling steps remain unchanged. Only a few of them are slightly modified and only a few additional steps are required. The main advantage of this approach is that it is a modular analysis technique, which allows to develop new features in the global modelling scheme simultaneously for a single dipole and a periodic array of dipoles. This is possible due to the fact that in both cases (for a single dipole and a periodic array of dipoles) the GFs in the spatial domain are calculated via the Inverse Fourier Transform (IFT) from the same spectral GFs. Fundamentally, the only difference is that the IFT is expressed in terms of double infinite integrals (single dipole) or a double infinite series (periodic array). The main problem in the numerical evaluation of the IFT is the poor convergence of the spectral components. The extraction technique allows to solve this problem. By subtracting the asymptotes from the spectral GFs and adding them again but to the spatial GFs, the numerically calculated part of the transform becomes much easier to handle. The main idea of our asymptote extraction technique is to use the same asymptotes in both cases (for a single dipole and a periodic array). At EUCAP 06 we formulated the requirements for these new asymptotes [6] and already demonstrated that they could be successfully used to model horizontal conductors. In this paper we report our progress in the implementation of vertical conductors. The combination of vertical and horizontal conductors widens the modelling horizon considerably.

It is also of interest to compare our approach with a direct approach. For periodic arrays the direct summation of the infinite spectral series remains rather popular because there are no problems with the branch point singularity and the surface waves poles that represent a considerable additional problem in the modelling of a single dipole.

The GFs are used in the MoM procedure to calculate the coupling matrices. If the direct approach is used, then in most cases the coupling matrices are calculated directly in the spectral domain, after which the IFT is used. The other option is to effectively calculate the spatial GFs and to calculate the coupling matrices from them in the spatial domain. From the theoretical point of view, the two algorithms are totally equivalent. However, from a numerical point of view there are very important differences. The direct approach requires the calculation of integrals over both a source and observation domain in order to obtain a corresponding element in the coupling matrix. Although these integrals can be calculated analytically in the spectral domain, which increases the convergence of the IFT, the integrals are expressed in terms of rather complex functions that require considerable numerical efforts to calculate them. Essentially, in our approach the calculation of the GFs can be expressed in terms of complex exponents. In many cases their calculation can be optimized so that only additions and multiplications of complex numbers are really necessary in the software routines, which makes the algorithm extremely efficient. The calculation of our coupling matrix elements is based on the expansion of the GFs in Taylor series and the analytical integration of the singularity in the source region.

The main application of our method is the modelling of frequency selective surfaces, textile materials with interlaced conductive strips, and periodic arrays.

\section{Theory}

Nowadays there are several methods that are used to construct electromagnetic solvers [3]. Probably, the most flexible ones in the description of the geometry are the solvers based on Finite Elements Method (FEM) and the Finite Difference Time Domain Method (FDTD). They are intrinsically capable 
to describe arbitrary geometries due to the meshing of the whole volume including the structure of interest. The changes of boundary conditions (periodic, waveguide or single antenna) can be switched without affecting the core of the solvers. These advantages make these solvers extremely popular. Other methods maybe not so flexible but they are efficient in the solution of specific problems. For instance, the method of moments (MoM) can deal very efficiently with horizontal conductors in planar layered media, scattering from conducting bodies and so on. The key advantage of the MoM is the use of Green's functions. Green's functions take into consideration the structure of the space where the antenna is located. As a consequence the number of unknowns is reduced to a number that is necessary to describe an electric current flowing on the conducting body. In most cases it is sufficient to use only a surface current which means that the total number of unknowns is much smaller than in the finite methods and it is easier to achieve a higher accuracy using much less unknowns. Unfortunately the application of this method requires a lot of analytical work that consists in the derivation of Green's function and the construction of the algorithm to their efficient calculation. The switch between different boundary conditions (periodic array-single antennawaveguide) is not also straightforward.

The application of the MoM procedure includes several steps. The first step consists in the introduction of an unknown equivalent current $J$, flowing on conducting bodies. Then, this current is approximated using a set of basis functions $f$ with unknown amplitudes.

$$
J=\sum_{q=1}^{N} A_{q} f_{q}
$$

Boundary conditions for tangential components of the fields on conducting bodies lead to an integral equations system

$$
E(J)+E^{i n c}=0
$$

Where $E(J)$ means the field excited by the current $J$ and $E^{i n c}$ stands for excitation fields. The testing reduces the integral equations system to a linear equations system

$$
Z \bullet A=P
$$

Where $Z_{q p}=\left\langle E\left(f_{q}\right), g_{p}\right\rangle ; P=\left\langle E^{i n c}, g_{p}\right\rangle$ and \langle\rangle stands for a scalar product and $g_{p}$ is the test function. The most difficult and time consuming part of the MoM is the calculation of all elements of the coupling matrix $\mathrm{Z}$.

For planar layered media the GFs and all fields components are known in a closed form in the spectral domain. Spectral components are the same for a single dipole and a periodic array of dipoles. The transform back to the spatial domain is performed via the IFT and it is expressed as double infinite integrals for a single dipole and as double series for a periodic array.
There are 2 approaches that are widely used in the construction of the MoM procedure depending on whether the primary testing is performed in the spectral domain or in the spatial domain. The difference consists in when the IFT is performed. If the testing is performed in the spectral domain then all integrations over the source and observation domain are performed in the spectral domain prior to the IFT. Normally all integrations can be performed analytically in terms of rather complex functions. Due to the double integration the convergence in the spectral domain is improved and singular points on the original path of integration can be avoided by modifying the contour of integrations if it is necessary. This approach is relatively easy to develop and to implement. However when the number of unknowns increases, the calculation time of the coupling matrix increases very fast. The efficiency can be improved by taking into account all types of symmetry but this step destroys the simplicity of the approach.

The second approach requires the calculation of Green's functions in the spatial domain at first. This is a quite complex task which requires a lot of additional work. The convergence of the spectral GFs is low and the source singularity should be extracted in an analytical way to obtain a stable algorithm. Then the integration over the source and observation domain is performed using the expansion of the GFs in Taylor series and the analytical integration of the singularity in the source region. This approach is evidently more complex but this is compensated so that in many cases it is more efficient in terms of the calculation time. The spectral GFs can be expressed in terms of complex exponents and in many cases the IFT can be optimized in such a way that only additions and multiplications of complex numbers are really necessary. KU Leuven uses the first approach only for test purposes. When the number of unknowns is small it is very efficient. The second approach becomes more efficient when the number of unknowns starts to grow.

Normally prior to the testing, the GFs are calculated on a grid of pre-selected points. These points are chosen to be equidistantly distributed over a rectangular area. Then an interpolation procedure is used to retrieve the value of the GFs in other points. The numerical integration is a relatively fast procedure due to the smoothness of the numerical GFs. Thus, the calculation time depends on the time needed to calculate the values of GFs in pre-selected points but not directly on the number of unknowns. This explains why the second approach becomes more efficient for structures with a larger number of unknowns.

KU Leuven has developed a MoM code to analyze single antennas in quasi 3D planar structures based on the second approach. The use of periodic structures creates the problem of how to incorporate periodicity in the most efficient way: whether it is better to start from zero or expand available models. Comparing available models with what is necessary to incorporate to model the periodicity, it was found that the periodicity can be implemented in the existing code for a single dipole rather quickly if it is possible to use the same asymptotic technique. It is necessary to change the routines that are responsible for the IFT and to introduce a new interpolation routine for two dimensional areas. The routines 
responsible for the calculation of the spectral GFs and for the testing remain almost unchanged.

In our paper presented [6] at EuCAP2006 asymptotes were introduced and the whole algorithm was successfully applied for horizontal conductors. For the reader's convenience. this asymptote extraction technique is briefly recalled. For a periodic array, the GFs are expressed in terms of the IFT as double series

$$
\begin{gathered}
G_{i j}=\sum_{k_{m x}} \sum_{k_{n y}} \widetilde{G}_{i j}\left(\beta_{m n}, z, z^{\prime}\right) e^{-j\left(k_{m x} x+k_{m y} y\right)}, \\
\beta=\sqrt{k_{m x}^{2}+k_{m y}^{2}}
\end{gathered}
$$

The poor convergence of the GFs in (4) is improved by subtracting specially selected asymptotes.

$$
\begin{gathered}
G F_{i j}=\sum_{m} \sum_{n}\left[\widetilde{G}_{i j}\left(\beta_{m n}\right)-\widetilde{G}_{i j}^{a s}\left(\beta_{m n}\right)\right] e^{-j\left(k_{m x} x+k_{m y} y\right)} \\
+\sum_{m} \sum_{n} g_{i j}^{a s}\left(r_{m n}\right)
\end{gathered}
$$

with $\sum_{m} \sum_{n} g_{i j}^{a s}\left(r_{m n}\right)=\sum_{m} \sum_{n} \widetilde{G}_{i j}^{a s}\left(\beta_{m n}\right) e^{-j\left(k_{m x} x+k_{m y} y\right)}$

In contrast to a single dipole, the second term in (5) is expressed in terms of double series imposing additional conditions that the asymptote in the spatial domain should also have a good convergence. The following asymptotes were selected:

$$
\widetilde{G}_{i j}^{a s}(\beta, t)=\frac{\left(1-e^{-\beta t}\right)^{m} e^{-\beta \Delta}}{\beta^{m}}
$$

The asymptote in (6) has the necessary leading term for large values of $\beta$, that annihilates the leading term of $\widetilde{G}_{i j}(\beta)$ and it has no singularity at $\beta=0$. The Fourier transform of (6) is known in a closed form.

$$
g^{a s}(r) \frac{1}{\sqrt{\rho+\Delta^{2}}}-\frac{1}{\sqrt{\rho^{2}+(\Delta+t)^{2}}},
$$

for $m=1$ and

$$
\begin{aligned}
& g^{a s}(x, y, z)=\ln \left[\frac{p_{1}(\rho, \Delta, t)}{p_{2}(\rho, \Delta) p_{3}(\rho, \Delta, t)}\right] \\
& p_{1}(\rho, \Delta, t)=\left(\sqrt{\rho^{2}+(\Delta+t)^{2}}+\Delta+t\right)^{2}, \\
& p_{2}(\rho, \Delta)=\left(\sqrt{\rho^{2}+\Delta^{2}}+\Delta\right) \\
& p_{3}(\rho, \Delta, t)=\sqrt{\rho^{2}+(\Delta+2 t)^{2}}+\Delta+2 t
\end{aligned}
$$

for $\mathrm{m}=2$.

The selected asymptotes possess the necessary convergence in the spectral and spatial domains. These asymptotes correspond to a static combined source of 2 anti-phased dipoles separated in the z-direction. In the spectral domain the convergence is ensured by the use of two dipoles and in the spatial domain the convergence in the horizontal plane is ensured by the phase shift between two dipoles. The asymptotes are relatively simple and it is possible to perform the integration over the source and observation domains. In general GFs for all interfaces should be calculated. For vertical conductors this leads to a very high number of GFs that should be stored. By integrating, the number of GFs needed to describe vertical conductors becomes proportional to the number of segments introduced on the vertical conductors.

The calculation of the series is performed using acceleration routines. The application of acceleration algorithms allows to reduce the number of terms in the series considerably. The series in the spectral domain are oscillating due to the exponential terms in (5). It is accelerated using Shank's transform [4]. The series in the spatial domain behave like a monotone series and they are accelerated using the rhoalgorithm [5] that it is more stable in this case. The general idea works very well, however there are some practical problems that require special attention.

The main problem is that it is unclear how many terms should be taken into account during the acceleration procedure to ensure stable results. Only, for the GFs associated with horizontal conductors, this problem is very well investigated [1]. In the case of GFs associated with vertical conductors the situation is a little bit more complex due to the fact that these GFs are expressed in terms of more complex functions. The threshold of the spectral parameter above which the leading asymptotic term becomes dominant depends on many factors. These factors are of interest in our ongoing research. The minimal number of terms in the series is about 10 by 10 . For the structures consisting only of horizontal strips this gives a good approximation.

\section{Numerical results}

One of the application areas of our model is the modelling of textiles with interlaced conducting fibres. These textiles can be described with some approximation as infinite periodic fibres consisting of vertical and horizontal conducting strips. The meshed strip is shown in Fig. 1. The size of a unit cell is $12 \mathrm{~mm}$ by $12 \mathrm{~mm}$ and the total height is about $3 \mathrm{~mm}$. The width of the strips is $1 \mathrm{~mm}$. The results are shown in Fig. 2 by a solid line. On the same figure by a dashed line the transmission through a periodic array of horizontal strips calculated using our approach is shown. The mesh was the same as in the first case. The last curve marked by diamond marks corresponds to lumped element models [2]. As expected, the slight deformation of the strips has a small effect on the transmission coefficient. The primary estimation 
of the shielding effectiveness of the textiles can be obtained using a simpler model with horizontal strips only.

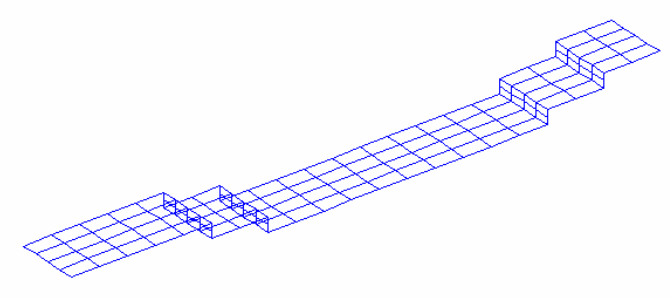

Fig. 1 Meshed infinite strip

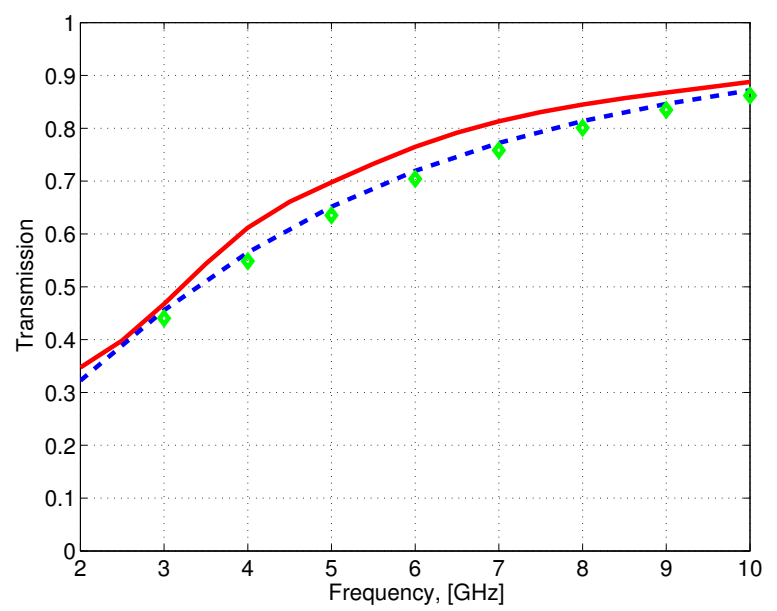

Fig. 2 Transmission through a periodic array of infinite strips

As a next example a finite step dipole is considered. The mesh is shown in Fig.3. The horizontal length of the dipole is $12 \mathrm{~mm}$ and the height of the step is $1 \mathrm{~mm}$. The size of a cell is $18 \mathrm{~mm}$ by $18 \mathrm{~mm}$. An array of these dipoles behaves like a frequency selective surface. The numerical results are plotted in Fig. 4 by a dashed line.

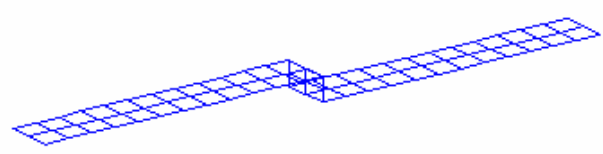

Fig. 3 Meshed step dipole

By a solid line the results obtained for a horizontal dipole without the vertical step are plotted. It can be observed again that a slight modification of the dipole leads to a small modification of the results. As expected, due to the fact that the total length of the dipole increases the resonance of the step dipole decreases.

The presented results illustrate that vertical conductors are implemented using the proposed technique.

Future work consists of the full testing of our software with structures that contain a complex combination of vertical and horizontal conductors and the implementation of volume currents.

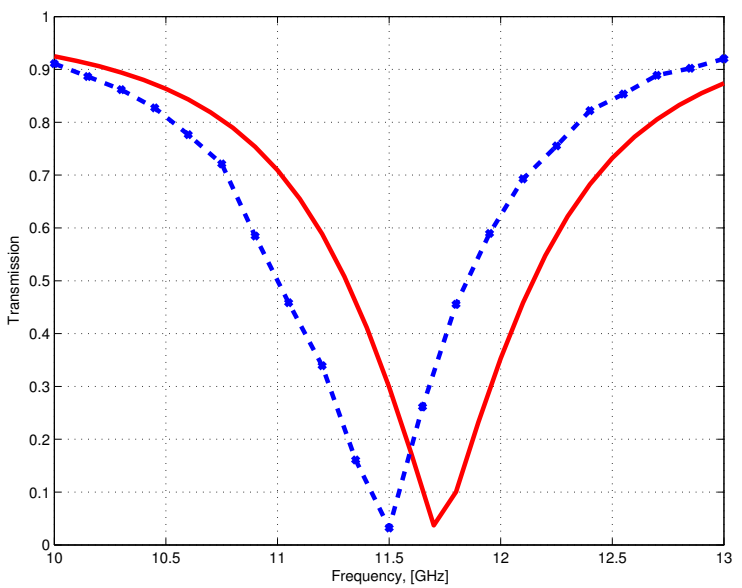

Fig. 4 Transmission through an array of finite dipoles

\section{Conclusions}

Using the asymptotic extraction technique proposed periodic arrays and single antennas can be modelled. Numerical results illustrate the success of this approach. This approach reduces the time needed to extend the capability of a software code from a single antenna to a periodic array.

\section{Acknowledgement}

KU Leuven is grateful to the IWT for the support of this work within the GBOU project "Predictive tools for permeability, mechanical and electro-magnetic properties of fibrous assemblies: modelling, simulation and experimental verification".

\section{References}

[1] P. Baccarelli et al., "Methods for the accelerated computation of Green's functions with 2-D periodicity in layered media," Proc. EuCAP 2006, Nice, France, 610 Nov. 2006

[2] N. Marcuvitz, "Waveguide Handbook", IEE Electromagnetic Waves Series, 1986

[3] A. F. Peterson, et al., «Computational Methods for Electromagnetics, IEEE Press, 1998

[4] D. Shanks, "Non-linear transformations of divergent and slowly convergent sequences," J. Math. Phys., vol. 34, pp. $1-42,1955$

[5] S. Singh, et al., "On the use of rho-algorithm in series acceleration", IEEE Antennas Propagation, vol. 39, 1514-1516, 1991

[6] V. Volski et al., "Efficient calculation of Green's functions for periodic arrays using a new asymptotic extraction technique", Proc. EuCAP 2006, Nice, France, 6-10 Nov. 2006 